\title{
Beta Error
}

National Cancer Institute

\section{Source}

National Cancer Institute. Beta Error. NCI Thesaurus. Code C142405.

In statistics, beta is the probability of a Type 2 error, when one incorrectly accepts the null hypothesis, creating a false negative. 